\title{
Truncations of $W_{\infty}$ Algebras
}

\author{
Mohammed Akram Fellah ${ }^{1}$ \\ ${ }^{1}$ Department of Applied Mathematics, University of Waterloo \\ \& Perimeter Institute for Theoretical Physics, Waterloo, Ontario, Canada \\ mafellah@uwaterloo.ca
}

We introduce a new class of Vertex Operator Algebras $Y^{+}$and their duals, which generalize the standard $\mathrm{W}$-algebras $W_{N}$ of type $\operatorname{sl}(N)$. These algebras can be defined in terms of junctions of boundary conditions and interfaces in the GL-twisted $N=4$ Super Yang Mills gauge theory. The aim of these technical calculations is to find the relation of these ortho-symplectic Y-algebras to truncations of even $W_{\infty}$. 


\section{Contents}

$1 \tilde{Y}^{+}$as even $\mathcal{W}_{\infty} \quad 1$

1.1 Parameters of $\tilde{Y}^{+} \quad 1$

1.2 Parameters of $Y^{+}$and their duals 2

1.3 Duality transformations 4

A Characters building blocks 4

\section{$1 \tilde{Y}^{+}$as even $\mathcal{W}_{\infty}$}

We expect relation of ortho-symplectic Y-algebras to truncations of even $\mathcal{W}_{\infty}$. These algebras contain roughly the same amount of generators as even $\mathcal{W}_{\infty}$ but extra, finite number of generators must be added. It would be nice to explore if one of the following happens:

- There exists a $Z_{2}$ projection that reduce ortho-symplectic Y-algebras to truncations of even $\mathcal{W}_{\infty}$

- Even $\mathcal{W}_{\infty}$ in general does not contain consistent truncations unless we add one or few extra fields.

- There is no relation between these two.

I suspect the first to be the case. Similar construction appeared in [13] to construct special classes of truncations of $\mathcal{N}=1 \mathcal{W}_{\infty}$. It would be nice to implement their argument here.

\subsection{Parameters of $\tilde{Y}^{+}$}

Recall definition of $\tilde{Y}^{+}$algebras

$$
\begin{aligned}
\tilde{Y}_{0, N, N}^{+}[\Psi] & =\frac{O S p(1 \mid 2 N)_{-\Psi+2 N+1} \times S b^{O S p(1 \mid 2 N)}}{S p(1 \mid 2 N)_{-\Psi+2 N+2}} \\
\tilde{Y}_{0, M, N}^{+}[\Psi] & =\frac{W_{2 N-2 M}\left[O S p(1 \mid 2 N)_{-\Psi+2 N+1}\right]}{O S p(1 \mid 2 M)_{-\Psi+2 M+2}}
\end{aligned}
$$


together with

$$
\begin{aligned}
\tilde{Y}_{L, 0,0}^{+}[\Psi] & =\frac{S O(2 L+1)_{-\Psi-2 L+1} \times F f^{S O(2 L+1)}}{S O(2 L+1)_{-\Psi-2 L+1}} \\
\tilde{Y}_{L, 0, N}^{+}[\Psi] & =\frac{W_{2 N}\left[O S p(2 L+1 \mid 2 N)_{-\Psi+2 N-2 L+1}\right]}{S O(2 L+1)_{-\Psi-2 L+2}} .
\end{aligned}
$$

We again assume that D3-branes can be removed if they recombine at the corner.

Central charge can be again written in factorized form $\mathrm{as}^{1}$

$$
c=\frac{1}{2}\left(\lambda_{1}+1\right)\left(\lambda_{2}+1\right)\left(\lambda_{3}+1\right)
$$

where

$$
\begin{aligned}
& \lambda_{1}=2 L-2 N(1-\Psi)-2 M \Psi \\
& \lambda_{2}=\frac{X}{\Psi-1} \\
& \lambda_{3}=-\frac{X}{\Psi} .
\end{aligned}
$$

These parameters again permute under the duality transformations.

Vacuum character can be most easily calculated for $\tilde{Y}_{L, 0, N}^{+}$. Characters of the other representations are conjecturally the same as their S-duals. Using formulas in the appendix one identifies the character with

$$
\tilde{\chi}_{L, 0, N}^{+}=\oint d V_{S O(2 L+1)} \chi_{D S}^{S p(2 N)} \chi_{F}[N, S O(2 L+1)]
$$

\subsection{Parameters of $Y^{+}$and their duals}

$Y^{+}$algebras are defined as

$$
\begin{aligned}
Y_{0, M, N}^{+}[\Psi] & =\frac{W_{2 N-2 M} S p(2 N)_{\frac{\Psi}{2}-N-1}}{S p(2 M)_{\frac{\Psi}{2}-M-\frac{3}{2}}} \quad N>M \\
Y_{L, 0,0}^{+}[\Psi] & =\frac{S O(2 L)_{-\frac{\Psi}{2}-L+1} \times F f S O(2 L)}{S O(2 L)_{-\frac{\Psi}{2}}+M-L+\frac{3}{2}} \\
Y_{L, 0, N}^{+}[\Psi] & =\frac{W_{2 N}\left[O S p(2 L \mid 2 N)_{-\frac{\psi}{2}+N-L+1}\right]}{S O(2 L)_{-\frac{\psi}{2}+N-L+\frac{3}{2}}} .
\end{aligned}
$$

\footnotetext{
${ }^{1}$ Note the \pm 1 difference compared to the unitary case.
} 
One can see it contains the cosets used in [6] (see also $[9,11]$ ). These algebras have dual construction in terms of

$$
\begin{aligned}
Y_{0, M, M}^{-}[\Psi] & =\frac{S O(2 M+1)_{-\Psi-2 M+2}}{S O(2 M)_{-\Psi-2 M+2}} \\
Y_{0, M, M+1}^{-}[\Psi] & =\frac{S O(2 M+2)_{-\Psi-2 M}}{S O(2 M+1)_{-\Psi-2 M}} \\
Y_{0, M, N}^{-}[\Psi] & =\frac{W_{2 N-2 M-1} S O(2 N)_{\psi-2 N+2}}{S O(2 M+1)_{\Psi-2 M}} \\
Y_{L, 0,0}^{-}[\Psi] & =\frac{O S p(1 \mid L)_{-\Psi+2 L+2}}{S p(2 L)_{-\Psi+2 L+2}} \\
Y_{L, 0, N}^{-}[\Psi] & =\frac{W_{2 N-1}\left[O S p(2 N \mid 2 L)_{\Psi+2 L+2}\right]}{O S p(1 \mid 2 L)_{\Psi+2 L}} .
\end{aligned}
$$

Central charges of these algebras are

$$
\begin{aligned}
c_{L, M, N}^{-}[\Psi]= & \tilde{c}_{L, N, M}^{-}[1-\Psi] \\
= & -\frac{(2(L-M)-1)(2(L-M)+1)(L-M)}{\Psi-1} \\
& \quad+\frac{2(2(L-N)+1)(L-N+1)(L-N)}{\Psi} \\
& +2 \Psi(2(M-N)+1)(M-N+1)(M-N) \\
& -2 L\left(1+6 M^{2}+M(6-12 N)-6 N+6 N^{2}\right) \\
& +4 M^{3}-3 M(1-2 N)^{2}+N\left(5-12 N+8 N^{2}\right) \\
c_{L, M, N}^{+}[\Psi]=- & \frac{2(M-L)(2(M-L)+1)(M-L+1)}{1-\Psi} \\
& -\frac{2(N-L)(2(N-L)+1)(N-L+1)}{\Psi} \\
+ & \Psi(2(M-N)-1)(2(M-N)+1)(M-N) \\
& +L\left(1-12(M-N)^{2}\right)-N+2(M-N)^{2}(3+2 M+4 N) .
\end{aligned}
$$

Calculation of vacuum characters goes in the same way as in the previous cases. Assuming the duality transformations that will be discussed below, we can restric only to the calculation of characters for $Y_{0, M, N}^{-}[\Psi]$. Vacuum character can be again described as

$$
\chi_{0, M, N}^{-}=\oint d V_{S O(2 L+1)} \chi_{D S}^{S O(2 N-2 L-1)} \chi_{B}[N-M, S O(2 M+1)]
$$




\subsection{Duality transformations}

Truncations given by algebra $\tilde{Y}^{+}$enjoy the same duality transformations as truncations of $\mathcal{W}_{\infty}$. There seem to be triality transformation on the parameter space of even $\mathcal{W}_{\infty}$ at least if we restrict to minimal models of this class.

In the case of $Y^{-}$there is no triality (or duality) action induced on the vertex operator algebras. On the other hand, there is a duality action maping above to two dual desctriptions given by

$$
Y_{L, M, N}^{-}[\Psi]=Y_{M, L, N}^{-}\left[\frac{1}{\Psi}\right]
$$

and

$$
Y_{L, M, N}^{+}[\Psi]=Y_{M, N, L}^{-}\left[1-\frac{1}{\Psi}\right]
$$

\section{A Characters building blocks}

Let us introduce following notation for different terms appearing in the calculation of characters of algebras in the main text

$$
\begin{aligned}
\chi_{D S}^{S O(2 N+1)} & =\chi_{D S}^{S p(2 N)}=\prod_{n=0}^{\infty} \prod_{i=1}^{N} \frac{1}{1-q^{2 i+n}} \\
\chi_{D S}^{S O(2 N)} & =\chi_{D S}^{S p(2 N)}=\prod_{n=0}^{\infty} \frac{1}{1-q^{N+n}} \prod_{i=1}^{N} \frac{1}{1-q^{2 i+n}}
\end{aligned}
$$

together with

$$
\begin{aligned}
\chi_{F}(N, S O(2 M+1)) & =\prod_{n=0}^{\infty} \frac{1}{1-q^{n+N+1}} \prod_{j=1}^{M} \frac{1}{1-x_{j} q^{n+N+1}} \frac{1}{1-x_{j}^{-1} q^{n+N+1}} \\
\chi_{F}(N, S O(2 M)) & =\chi_{F}(N, S p(2 M))=\prod_{n=0}^{\infty} \prod_{j=1}^{M} \frac{1}{1-x_{j} q^{n+N+1}} \frac{1}{1-x_{j}^{-1} q^{n+N+1}} \\
\chi_{B}(N, S O(2 M+1)) & =\prod_{n=0}^{\infty}\left(1+q^{n+N+1}\right) \prod_{j=1}^{M}\left(1+x_{j} q^{n+N+1}\right)\left(1+x_{j}^{-1} q^{n+N+1}\right) \\
\chi_{B}(N, S O(2 M)) & =\chi_{B}(N, S p(2 M))=\prod_{n=0}^{\infty} \prod_{j=1}^{M}\left(1+x_{j} q^{n+N+1}\right)\left(1+x_{j}^{-1} q^{n+N+(1} \mathrm{A} .2\right)
\end{aligned}
$$


and the measure given by

$$
\oint d V_{S O(2 L+1)}=\frac{1}{2^{L} L !} \oint \prod_{i=1}^{L} \frac{d x_{i}}{x_{i}} \prod_{i=1}^{L}\left[( 1 - x _ { i } ) ( 1 - x _ { i } ^ { - 1 } ) \prod _ { j = i + 1 } ^ { L } ( 1 - x _ { i } x _ { j } ) ( 1 - x _ { i } ^ { - 1 } x _ { j } ) ( 1 - x _ { i } x _ { j } ^ { - 1 } ) \left(1-x_{i}^{-1} x_{j}^{-1}\right.\right.
$$

\section{References}

[1] M. R. Gaberdiel and R. Gopakumar, "Triality in Minimal Model Holography," JHEP 1207, 127 (2012) doi:10.1007/JHEP07(2012)127 [arXiv:1205.2472 [hep-th]].

[2] T. Procházka, "Exploring $\mathcal{W}_{\infty}$ in the quadratic basis," JHEP 1509, 116 (2015) doi:10.1007/JHEP09(2015)116 [arXiv:1411.7697 [hep-th]].

[3] T. Procházka, "W -symmetry, topological vertex and affine Yangian," JHEP 1610, 077 (2016) doi:10.1007/JHEP10(2016)077 [arXiv:1512.07178 [hep-th]].

[4] D. Gaiotto and M. Rapčák, "Vertex Algebras at the Corner," arXiv:1703.00982 [hep-th].

[5] A. Kapustin and E. Witten, Commun. Num. Theor. Phys. 1, 1 (2007) doi:10.4310/CNTP.2007.v1.n1.a1 [hep-th/0604151].

[6] M. R. Gaberdiel and C. Vollenweider, "Minimal Model Holography for SO(2N)," JHEP 1108, 104 (2011) doi:10.1007/JHEP08(2011)104 [arXiv:1106.2634 [hep-th]].

[7] C. Candu and M. R. Gaberdiel, "Supersymmetric holography on $A d S_{3}$," JHEP 1309, 071 (2013) doi:10.1007/JHEP09(2013)071 [arXiv:1203.1939 [hep-th]].

[8] C. Candu and M. R. Gaberdiel, "Duality in N=2 Minimal Model Holography," JHEP 1302, 070 (2013) doi:10.1007/JHEP02(2013)070 [arXiv:1207.6646 [hep-th]].

[9] C. Candu, M. R. Gaberdiel, M. Kelm and C. Vollenweider, "Even spin minimal model holography," JHEP 1301, 185 (2013) doi:10.1007/JHEP01(2013)185 [arXiv:1211.3113 [hep-th]].

[10] M. Beccaria, C. Candu, M. R. Gaberdiel and M. Groher, "N=1 extension of minimal model holography," JHEP 1307, 174 (2013) doi:10.1007/JHEP07(2013)174 [arXiv:1305.1048 [hep-th]].

[11] M. Beccaria, C. Candu and M. R. Gaberdiel, "The large N $=4$ superconformal $W_{\infty}$ algebra," JHEP 1406, 117 (2014) doi:10.1007/JHEP06(2014)117 [arXiv:1404.1694 [hep-th]].

[12] K. Ferreira, "Even spin $\mathcal{N}=4$ holography," arXiv:1702.02641 [hep-th].

[13] C. Candu and C. Vollenweider, "The $\mathcal{N}=1$ algebra $\mathcal{W}_{\infty}[\mu]$ and its truncations," JHEP 1311, 032 (2013) doi:10.1007/JHEP11(2013)032 [arXiv:1305.0013 [hep-th]]. 
[14] T. Creutzig, Y. Hikida and P. B. Rnne, "N $\mathcal{N}=1$ supersymmetric higher spin holography on $A d S_{3}$," JHEP 1302, 019 (2013) doi:10.1007/JHEP02(2013)019 [arXiv:1209.5404 [hep-th]]. 